Litman, B. J. (1973) Biochemistry 12, 2545-2554.

Lowry, O. H., Rosebrough, N. J., Farr, A. L., \& Randall, R. J. (1951) J. Biol. Chem. 193, 265-275.

Mimms, L. T., Zampighi, G., Nazaki, Y., Tanford, C., \& Reynolds, J. A. (1981) Biochemistry 20, 833-840.

Noller, C. R., \& Rockwell, W. C. (1938) J. Am. Chem. Soc. 60, 2076-2077.

O'Brien, D. F., Costa, L. F., \& Ott, R. A. (1977) Biochemistry 16, 1295-1303.

Papermaster, D. S., \& Dreyer, W. J. (1974) Biochemistry 13, 2438-2444.

Petri, W. A., Jr., \& Wagner, R. R. (1979) J. Biol. Chem. 254, 4313-4316.

Racker, E. (1979) Methods Enzymol. 55, 699-711.

Razin, S. (1972) Biochim. Biophys. Acta 265, 241-296.
Roseman, M. A., Holloway, P. W., Calabro, M. A., \& Thompson, T. E. (1977) J. Biol. Chem. 252, 4842-4849. Shinoda, K., Yamaguchi, T., \& Hori, R. (1961) Bull. Chem. Soc. Jpn. 34, 237-241.

Smith, H. G., Jr., Stubbs, G. W., \& Litman, B. J. (1975) Exp. Eye Res. 20, 211-217.

Spiro, R. G. (1966) Methods Enzymol. 8, 3-26.

Steele, J. C. H., Jr., Tanford, C., \& Reynolds, J. A. (1978) Methods Enzymol. 48, 11-23.

Stubbs, G. W., \& Litman, B. J. (1978) Biochemistry 17, 215-219.

Stubbs, G. W., Smith, H. G., Jr., \& Litman, B. J. (1976) Biochim. Biophys. Acta 426, 46-56.

Watts, A., Volotovski, I. D., \& Marsh, D. (1979) Biochemistry 18, 5006-5013.

\title{
Size Dependence of the Translational Diffusion of Large Integral Membrane Proteins in Liquid-Crystalline Phase Lipid Bilayers. A Study Using Fluorescence Recovery after Photobleaching ${ }^{\dagger}$
}

\author{
Winchil L. C. Vaz,* Manuel Criado, Vitor M. C. Madeira, Guenther Schoellmann, and Thomas M. Jovin
}

ABSTRACT: The translational diffusion of bovine rhodopsin, the $\mathrm{Ca}^{2+}$-activated adenosinetriphosphatase of rabbit muscle sarcoplasmic reticulum, and the acetylcholine receptor monomer of Torpedo marmorata has been examined at a high dilution (molar ratios of lipid/protein $\geq 3000 / 1$ ) in liquidcrystalline phase phospholipid bilayer membranes by using the fluorescence recovery after photobleaching technique. These integral membrane proteins having molecular weights of about 37000 for rhodopsin, about 100000 for the adenosinetriphosphatase, and about 250000 for the acetylcholine receptor were reconstituted into membranes of dimyristoylphosphatidylcholine (rhodopsin and acetylcholine receptor), soybean lipids (acetylcholine receptor), and a total lipid extract of rabbit muscle sarcoplasmic reticulum (adenosinetriphosphatase). The translational diffusion coefficients of all the proteins at $310 \mathrm{~K}$ were found to be in the range $(1-3) \times$ $10^{-8} \mathrm{~cm}^{2} / \mathrm{s}$. In consideration of the sizes of the membranebound portions of these proteins, this result is in agreement

$I_{1}$ n recent years considerable effort has been dedicated to the study of the diffusion of membrane components in model phospholipid bilayer membranes [for reviews see Cherry (1979), Jovin et al. (1981), and Vaz et al. (1982)]. The purpose of such model membrane studies is primarily to understand the diffusion of membrane components in terms of

\footnotetext{
${ }^{\dagger}$ From the Max-Planck-Institut für biophysikalische Chemie, D-3400 Göttingen-Nikolausberg, FRG (W.L.C.V., M.C., and T.M.J.), the Departamento de Zoologia, Universidade de Coimbra, 3049 Coimbra Codex, Portugal (V.M.C.M.), and the Departments of Biochemistry and Ophthalmology, Tulane University School of Medicine, New Orleans, Louisiana 70112 (G.S.). Received May 20, 1982. M.C. is holder of a postdoctoral fellowship from the Alexander von Humboldt Stiftung, Bonn-Bad Godesberg, FRG, and V.M.C.M. received a travel grant from the Fundação Calouste Gulbenkian, Lisboa, Portugal. This work was supported in part by Research Grant EY03350 (to G.S.) from the National Eye Institute.
}

with the weak dependence of the translational diffusion coefficient upon diffusing particle size predicted by continuum fluid hydrodynamic models for the diffusion in membranes [Saffman, P. G., \& Delbrück, M. (1975) Proc. Natl. Acad. Sci. U.S.A. 72, 3111-3113]. Lipid diffusion was also examined in the same lipid bilayers with the fluorescent lipid derivative $N$-(7-nitro-2, 1,3-benzoxadiazol-4-yl)dimyristoylphosphatidylethanolamine. The translational diffusion coefficient for this lipid derivative was found to be in the range $(9-14) \times 10^{-8} \mathrm{~cm}^{2} / \mathrm{s}$ at $310 \mathrm{~K}$. In consideration of the dimensions of the lipid molecule, this value for the lipid diffusion coefficient is in agreement with the continuum fluid hydrodynamic model only if a near-complete slip boundary condition is assumed at the bilayer midplane. Alternatively, kinetic diffusion models [Träuble, H., \& Sackmann, E. (1972) J. Am. Chem. Soc. 94, 4499-4510] may have to be invoked to explain the lipid diffusion behavior.

the physical parameters governing such diffusion in the quasi-two-dimensional lipid bilayer matrix and, eventually, to have a base line to which diffusion studies in the far more complex biological membranes may be compared.

Recent studies on the translational diffusion of membrane-bound peptides and proteins (Wu et al., 1978; Smith et al., 1979a, 1980; Vaz et al., 1979, 1981) in reconstituted model membrane systems have indicated that the diffusion of these entities in liquid-crystalline phase phospholipid bilayers is rapid, with translational diffusion coefficients $\left(D_{t}\right)^{1}$ in the

\footnotetext{
'Abbreviations: AchR, acetylcholine receptor from Torpedo marmorata; DMPC, dimyristoylphosphatidylcholine; $D_{1}$, translational diffusion coefficient: FRAP, fluorescence recovery after photobleaching; NBD-DMPE, $N$-(7-nitro-2,1,3-benzoxadiazol-4-yl)dimyristoylphosphatidylethanolamine; SR-ATPase, the $\mathrm{Ca}^{2+}$-activated adenosinetriphosphatase from rabbit skeletal muscle sarcoplasmic reticulum.
} 
range $(1-10) \times 10^{-8} \mathrm{~cm}^{2} / \mathrm{s}$. In these studies, however, the range of protein sizes examined was quite small and no meaningful conclusions regarding the dependence of protein diffusion upon size were possible. In this paper we report our results on the diffusion behavior of large integral membrane proteins in reconstituted lipid bilayer membranes in the liquid-crystalline phase. The proteins examined were bovine rhodopsin $\left(M_{\mathrm{r}} 37000\right)$, SR-ATPase of rabbit skeletal muscle sarcoplasmic reticulum $\left(M_{\mathrm{r}} 100000\right)$, and AchR from Torpedo marmorata in its monomeric form $\left(M_{\mathrm{r}} 250000\right)$. The size range of the proteins examined here allows a meaningful evaluation of the diffusion behavior of integral membrane proteins as a function of their size. From values for the dimensions of these proteins available in the literature (Yeager, 1976; Osborne et al., 1978; Fleischer et al., 1979; Brady et al., 1981; Wise et al., 1979), the values of $D_{\mathrm{t}}$ plotted as a function of protein radii are in reasonably good agreement with the model of Saffman and Delbrück (Saffman, 1976; Saffman \& Delbrück, 1975) for diffusion in thin viscous fluid sheets.

\section{Materials and Methods}

Reagents and Proteins. Fluorescein isothiocyanate and fluorescein iodoacetamide were from Molecular Probes, Plano, TX, DMPC was from Fluka AG, Buchs, Switzerland, soybean lipids (soybean phosphatidylcholine, type II-S) and octyl glucoside were from Sigma Chemie, Munich, FRG, and sodium cholate was from E. Merck, Darmstadt, FRG. All of the above chemicals and lipids were used as received. A total lipid extract of rabbit muscle sarcoplasmic reticulum was prepared as described by Madeira \& Antunes-Madeira (1976). NBD-DMPE was a gift from Dr. D. Hallmann, MaxPlanck-Institut, Göttingen, FRG. Bovine rhodopsin was purified from bovine rod outer segment disks by affinity chromatography on concanavalin A-Sepharose (Pharmacia Fine Chemicals, Uppsala, Sweden) in the presence of octyl glucoside as described by Albert \& Litman (1978). SR-ATPase was prepared as described by Almeida et al. (1982). Purification of AchR as the monomer has been described in detail elsewhere (Criado et al., 1982). Rhodopsin in an octyl glucoside micellar solution and SR-ATPase in sarcoplasmic reticulum vesicles from which all other proteins except the SR-ATPase were stripped (Almeida et al., 1982) were labeled with fluorescein iodoacetamide by using a $1 / 10$ molar ratio of protein to dye reagent in aqueous buffers at $\mathrm{pH} 7-8$. The labeling ratios were about two dye molecules per protein molecule in both cases. The Ach R monomer was labeled with fluorescein isothiocyanate at a $\mathrm{pH}$ of 9.2 to give a dye/protein ratio of about $19 / 1$. In spite of this high labeling ratio no effect upon the binding of $\alpha$-bungarotoxin by the labeled protein relative to unlabeled protein was observed (Criado et al., 1982).

Reconstitution of Proteins into Lipid Bilayers. The reconstitution of purified and fluorescein-labeled proteins was done by detergent dialysis to give dilute proteoliposome suspensions. The lipid/protein molar ratios were about 3000/1 for rhodopsin, 5000/1 for SR-ATPase, and about 10000/1 for AchR monomer. The detergents used in the dialysis were octyl glucoside for rhodopsin and SR-ATPase and cholate for AchR. Under the conditions used in this work, the molar ratio of residual detergent to lipid was less than $1 / 160$. Slides for FRAP experiments were prepared essentially as described earlier (Vaz et al., 1981) taking care not to exceed $35^{\circ} \mathrm{C}$ in any of the steps. In the case of rhodopsin all steps were carried out in the dark, and the slides were exposed to daylight, thus bleaching the rhodopsin, only just before doing the FRAP experiments. It was often observed that the fluorescent-labeled proteins formed intensely fluorescent patches which showed

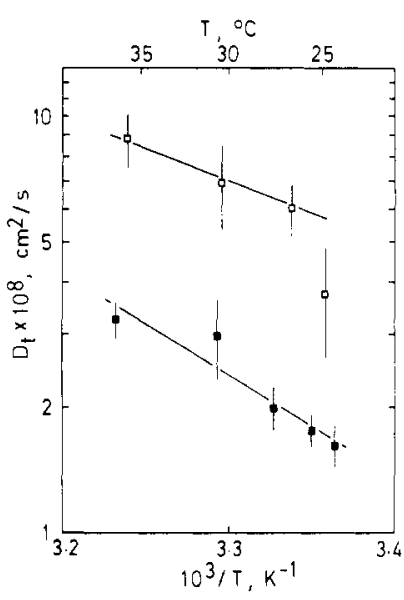

FIGURE 1: Arrhenius plots of the translational diffusion of bleached rhodopsin $(\square)$ and NBD-DMPE ( $\square$ ) in multibilayers of DMPC. Each experimental point in this and other figures is the mean \pm standard deviation (indicated as vertical bars) from at least five FRAP experiments. The lines are linear regression least-squares analyses of the experimental points. The coefficients of correlation were 0.95 and 0.97 for the protein and lipid data, respectively. The NBD-DMPE data were taken in the temperature range $25-59^{\circ} \mathrm{C}$, and the analysis is for the data above $25^{\circ} \mathrm{C}$.

no recovery of fluorescence after photobleaching even when the lipid was in the liquid-crystalline phase. Some combinations of protein and lipid always showed these patches while others did not. Samples that showed this sort of patching were not used in the FRAP experiments.

FRAP Measurements. The FRAP apparatus has been described in detail elsewhere (Criado et al., 1982). The illuminated area had a Gaussian intensity profile with a $1 / e^{2}$ radius of $5.3 \mu \mathrm{m}$. $D_{\mathrm{t}}$ was derived from fluorescence recovery curves by using the half-times for complete fluorescence recovery as described by Axelrod et al. (1976). Some experimental curves were compared with theoretical recovery curves for recovery due to redistribution of one diffusing component. The agreement between experiment and theory was fairly good when the lipid bilayers were in their liquid-crystalline phase. In all cases the fluorescence recovery at "infinite" time after photobleaching was $\geq 95 \%$ of the fluorescence intensity prior to photobleaching.

Results

Rhodopsin Diffusion in Liquid-Crystalline Phase DMPC. The translational diffusion of bovine rhodopsin (in the bleached form) was studied in multibilayers of DMPC in the liquidcrystalline phase at a lipid/protein molar ratio of about $3000 / 1$. The temperature dependence of $D_{t}$ is shown as an Arrhenius plot in Figure $1 . D_{\text {t }}$ drops monotonically from a value of $(3.3 \pm 0.3) \times 10^{-8} \mathrm{~cm}^{2} / \mathrm{s}$ at about $36^{\circ} \mathrm{C}$ to a value of $(1.6 \pm 0.2) \times 10^{-8} \mathrm{~cm}^{2} / \mathrm{s}$ at about $24^{\circ} \mathrm{C}$, showing an apparent activation energy of $46.9 \mathrm{~kJ} / \mathrm{mol}\left(24-36{ }^{\circ} \mathrm{C}\right)$. Diffusion of NBD-DMPE in DMPC multibilayers (Figure 1) also shows a monotonic drop in $D_{t}$ from a value of $(8.8 \pm 1.3)$ $\times 10^{-8} \mathrm{~cm}^{2} / \mathrm{s}$ at about $36^{\circ} \mathrm{C}$ to a value of $(3.7 \pm 1.1) \times 10^{-8}$ $\mathrm{cm}^{2} / \mathrm{s}$ at about $25^{\circ} \mathrm{C}$. In this case the apparent activation energy is $28.5 \mathrm{~kJ} / \mathrm{mol}\left(25-59^{\circ} \mathrm{C}\right)$.

SR-ATPase Diffusion in Liquid-Crystalline Phase Bilayers of a Total Lipid Extract of the Sarcoplasmic Reticulum. In our hands successful reconstitution of the SR-ATPase in terms of the criteria described under Materials and Methods was possible only in multibilayers and liposomes of a total lipid extract of the sarcoplasmic reticulum. DMPC and egg phosphatidylcholine were tried without success. The total lipid extract of sarcoplasmic reticulum is a mixture of phospholipids 


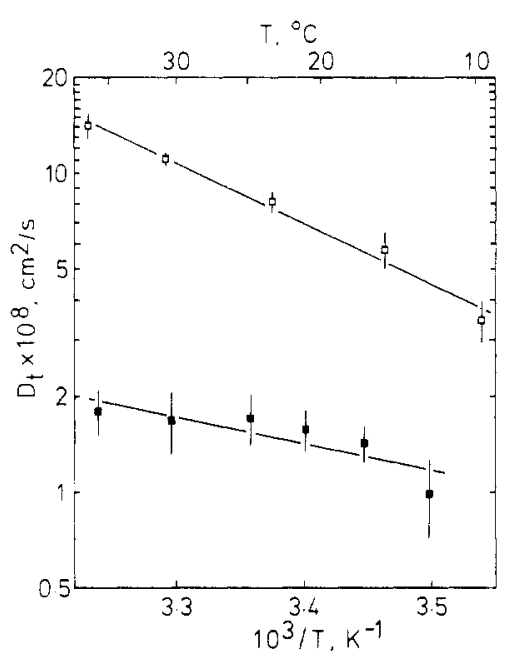

FIGURE 2: Arrhenius plots for the translational diffusion of SR-ATPase $(\boldsymbol{\omega})$ and NBD-DMPE (a) in multibilayers of a total lipid extract of sarcoplasmic reticulum. The lines are linear regression least-squares analyses of the experimental data. The coefficients of correlation were 0.85 and 0.99 for the SR-ATPase data and the NBD-DMPE data, respectively.

containing mainly phosphatidylcholine, with small amounts of phosphatidylethanolamine and other minor lipids (Madeira \& Antunes-Madeira, 1976). The translational diffusion of SR-ATPase reconstituted into bilayers of total sarcoplasmic reticulum lipids at a molar ratio of lipid/protein of about $5000 / 1$ was studied in the temperature range between 13 and $36^{\circ} \mathrm{C}$. No phase transitions occur in bilayers of this lipid in this range of temperature. The results of the FRAP experiments are seen in Figure 2. A monotonic dependence of protein $D_{\mathrm{t}}$ is observed with an activation energy of $16.2 \mathrm{~kJ} / \mathrm{mol}$ $\left(13-36{ }^{\circ} \mathrm{C}\right) . \quad D_{\mathrm{t}}$ has values of $(1.8 \pm 0.3) \times 10^{-8} \mathrm{~cm}^{2} / \mathrm{s}$ at about $36^{\circ} \mathrm{C}$ and $(9.9 \pm 2.7) \times 10^{-9} \mathrm{~cm}^{2} / \mathrm{s}$ at about $13^{\circ} \mathrm{C}$. The results for NBD-DMPE diffusion in the same system are also seen in Figure 2. Lipid probe $D_{\mathrm{t}}$ shows a monotonic temperature dependence with an activation energy of 36.7 $\mathrm{kJ} / \mathrm{mol}$, dropping from $(1.4 \pm 0.1) \times 10^{-7} \mathrm{~cm}^{2} / \mathrm{s}$ at about 37 ${ }^{\circ} \mathrm{C}$ to $(3.5 \pm 0.5) \times 10^{-8} \mathrm{~cm}^{2} / \mathrm{s}$ at about $10^{\circ} \mathrm{C}$.

AchR Monomer Diffusion in Liquid-Crystalline Phase Multibilayers of DMPC and Soybean Lipids. A detailed report of the translational diffusion behavior of the AchR protein in its monomeric and dimeric forms in several lipid systems has been reported elsewhere (Criado et al., 1982). Here we shall consider only the results for diffusion of the monomeric protein in multibilayers of DMPC and soybean lipids. The temperature dependence of $D_{\mathrm{t}}$ for this protein in these lipid bilayers is shown as Arrhenius plots in Figure 3. In liquid-crystalline DMPC, $D_{t}$ changes monotonically from a value of $(2.4 \pm 0.8) \times 10^{-8} \mathrm{~cm}^{2} / \mathrm{s}$ at about $36^{\circ} \mathrm{C}$ to a value of $(1.6 \pm 0.3) \times 10^{-8} \mathrm{~cm}^{2} / \mathrm{s}$ at about $27^{\circ} \mathrm{C}$. The apparent activation energy is $33.5 \mathrm{~kJ} / \mathrm{mol}\left(27-36^{\circ} \mathrm{C}\right)$. In soybean lipid multibilayers, too, $D_{\mathrm{t}}$ for the protein drops monotonically from $(3.3 \pm 0.7) \times 10^{-8} \mathrm{~cm}^{2} / \mathrm{s}$ at about $37^{\circ} \mathrm{C}$ to $(1.5 \pm 0.2) \times 10^{-8}$ $\mathrm{cm}^{2} / \mathrm{s}$ at about $15^{\circ} \mathrm{C}$. The apparent activation energy is 25.0 $\mathrm{kJ} / \mathrm{mol}\left(15-37^{\circ} \mathrm{C}\right)$. This may be compared with the diffusion of NBD-DMPE in DMPC multibilayers (Figure 1) and in soybean lipid multibilayers (Figure 3 ). In the latter lipid, $D_{\mathrm{t}}$ for NBD-DMPE drops monotonically from $(1.1 \pm 0.1) \times 10^{-7}$ $\mathrm{cm}^{2} / \mathrm{s}$ at about $37^{\circ} \mathrm{C}$ to $(3.4 \pm 0.6) \times 10^{-8} \mathrm{~cm}^{2} / \mathrm{s}$ at about $15^{\circ} \mathrm{C}$, showing an apparent activation energy of $36.8 \mathrm{~kJ} / \mathrm{mol}$ $\left(15-37^{\circ} \mathrm{C}\right)$.

Over the temperature ranges examined in this work, the diffusion behavior of proteins and lipids appears to obey the Arrhenius law (with apparent activation energies between

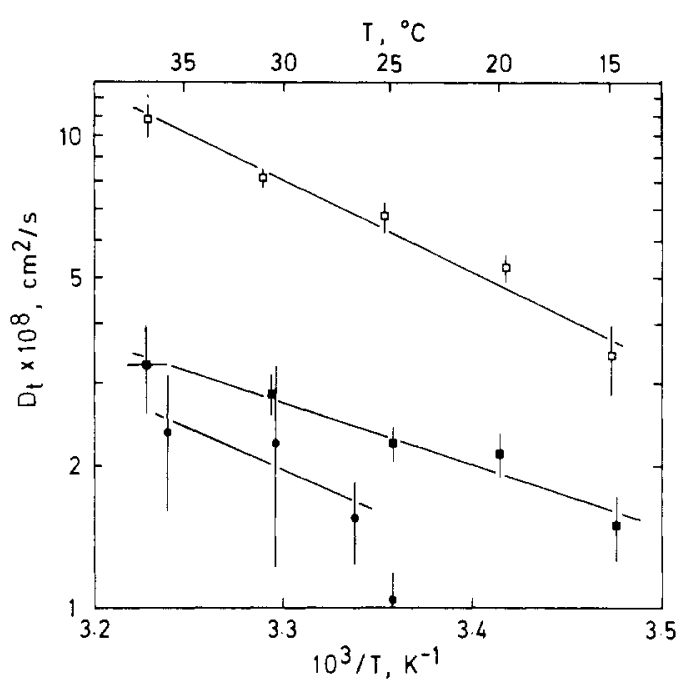

FIGURE 3: Arrhenius plots for the translational diffusion of the AchR monomer in multibilayers of DMPC $(\bullet)$, soybean lipids $(\square)$, and NBD-DMPE in soybean lipids ( $\square$ ). The lines are linear regression least-squares analyses of the experimental points. Coefficients of correlation were $0.87,0.98$, and 0.99 for the AchR data in DMPC and soybean lipids and the NBD-DMPE data in soybean lipids, respectively.

about 16 and about $47 \mathrm{~kJ} / \mathrm{mol}$ ). There is, however, no obvious correlation between the apparent activation energies and the nature of the diffusant or the bilayer system being investigated.

\section{Discussion}

We shall first interpret the protein diffusion results presented in the preceding section in terms of the sizes of the diffusing entities. Neutron and X-ray scattering studies on rhodopsin (Yeager, 1976; Osborne et al., 1978), the SR-ATPase (Fleischer et al., 1979; Brady et al., 1981), and the AchR monomer (Wise et al., 1979) provide information on the sizes and shapes of these proteins. According to these studies, the shape of rhodopsin in detergent micellar solutions may be approximated to a prolate ellipsoid with dimensions of $9.0 \times$ $4.5 \times 4.5 \mathrm{~nm}$ (Yeager, 1976) or to a cylinder with a height and radius of 7.0 and $1.7 \mathrm{~nm}$, respectively (Osborne et al., 1978). If the thickness of the lipid bilayer is taken to be 5.0 $\mathrm{nm}$, the membrane-bound portion of this protein may be considered to be roughly cylindrical with a height of $5.0 \mathrm{~nm}$ (equal to the membrane thickness) and a radius of $1.7-2.3 \mathrm{~nm}$. The dimensions of this protein in membranes are probably the same as in detergent micelles (Yeager et al,, 1980). For the SR-ATPase in detergent micellar solutions, $\mathrm{X}$-ray scattering studies suggest the protein to have a cylindrical shape with a height and radius of 14.2 and $3.5 \mathrm{~nm}$, respectively (Brady et al., 1981). In detergent micelles the protein is in the monomeric state. In reconstituted membranes, however, this protein appears to form an oligomer (two to four monomers) with an average diameter of about $6.0 \mathrm{~nm}$ in the plane of the membrane (Fleischer et al., 1979). Finally, neutron scattering results on the AchR monomer in detergent micellar solutions have been interpreted in terms of an asymmetric dumbbell shape for this protein with a cylindrical membrane-bound portion having a height of $5.5 \mathrm{~nm}$ and a radius of $1.5 \mathrm{~nm}$ (Wise et al., 1979).

The diffusion data may now be plotted as a function of protein radius as shown in Figure 4. It has been assumed here that the frictional drag forces exerted upon the hydrophilic portions of the proteins extending into the aqueous phase are negligible in comparison with those exerted upon the membrane-bound portions of these proteins. Experimental support 


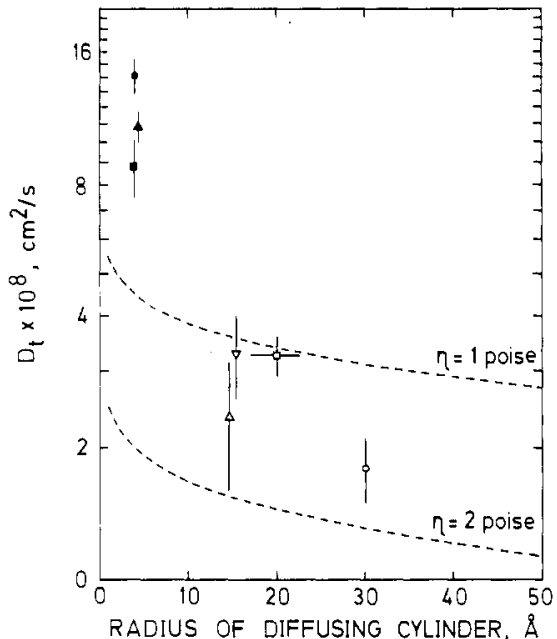

FIGURE 4: Dependence of the experimentally obtained $D_{t}$ of the proteins and lipid probes studied in this work upon the radius of the diffusant. The data are for bleached rhodopsin in DMPC multibilayers at $36^{\circ} \mathrm{C}(\square)$, the SR-ATPase in multibilayers of a total lipid extract of rabbit muscle sarcoplasmic reticulum at $36^{\circ} \mathrm{C}(0)$, the AchR monomer in soybean lipid multibilayers at $37^{\circ} \mathrm{C}(\nabla)$, and the AchR monomer in DMPC multibilayers at $36^{\circ} \mathrm{C}(\Delta)$. Lipid diffusion was measured by using the lipid probe, NBD-DMPE, in multibilayers of DMPC at $36^{\circ} \mathrm{C}(\boldsymbol{\square})$, of soybean lipids at $37^{\circ} \mathrm{C}(\mathbf{\Delta})$, and of a total lipid extract of sarcoplasmic reticulum at $37^{\circ} \mathrm{C}(\boldsymbol{)})$. The horizontal bar at the experimental point for bleached rhodopsin gives the limits of the radius of this protein as a prolate ellipsoid ( $2.3 \mathrm{~nm}$; Yeager, 1976) or as a cylinder ( $1.7 \mathrm{~nm}$; Osborne et al., 1978). The lipid probe, NBD-DMPE, was assumed to diffuse as a cylinder of radius $0.4 \mathrm{~nm}$. The broken lines are the translational diffusion coefficients as a function of particle radius for assumed membrane viscosities of 1 and $2 \mathrm{P}$, predicted by eq 1 . The theoretical curves were computed for $T=310 \mathrm{~K}, h=5.0 \mathrm{~nm}$, and $\eta^{\prime}=0.01 \mathrm{P}$

of this assumption was provided by Smith et al. (1979b). For the purposes of Figure 4, therefore, we have treated all proteins as cylinders with heights of $5.0 \mathrm{~nm}$ (the thickness of the lipid bilayer) and radii derived from the neutron and $X$-ray scattering results reported in the literature. It is seen that $D_{\mathrm{t}}$ is a relatively insensitive function of the protein radius. Such a weak dependence is predicted by the continuum hydrodynamic model for diffusion in thin viscous fluid sheets presented by Saffman and Delbrück (Saffman, 1976; Saffman \& Delbrück, 1975). These authors give $D_{\mathrm{t}}$ as a function of radius to be

$$
D_{\mathrm{t}}=\frac{k T}{4 \pi \eta h}\left[\ln \left(\frac{\eta h}{\eta^{\prime} a}\right)-\gamma\right]
$$

Here $k$ is Boltzmann's constant, $T$ is the absolute temperature, $\eta$ is the viscosity of the fluid constituting the sheet, $\eta^{\prime}$ is the viscosity of the bounding fluid on both sides of the sheet (assumed to be very much smaller than $\eta$ ), $h$ is the height of the cylindrical particle (also the thickness of the sheet), $a$ is the radius of the particle, and $\gamma$ is Euler's constant (0.5772). In Figure 4 we have included the theoretical predictions of this model for assumed membrane viscosities of 1.0 and $2.0 \mathrm{P}$. The protein diffusion data reported here agree with these limits for membrane viscosity. In the recent literature, values of $D_{\mathrm{t}}$ for other large proteins reconstituted into liquid-crystalline phase phospholipid bilayers have been reported (Peters \& Cherry, 1982; Chang et al., 1981). Peters \& Cherry (1982) have reported $D_{\mathrm{t}}$ for bacteriorhodopsin to be $2.3 \times 10^{-8} \mathrm{~cm}^{2} / \mathrm{s}$ at $32{ }^{\circ} \mathrm{C}$ in DMPC bilayers at a lipid/protein molar ratio of $140 / 1$ (which is not in keeping with the high dilution criterion that we have used in our work). With values of 1.6 and 4.5 $\mathrm{nm}$ for the radius and height, respectively, of bacterio- rhodopsin, and assuming the validity of the Saffman-Delbrück model for diffusion in membranes, these workers calculate a membrane viscosity of $1.8 \mathrm{P}$ for the DMPC bilayer at $32^{\circ} \mathrm{C}$. Chang et al. (1981) have studied the translational diffusion of erythrocyte band 3 in bilayers of DMPC. $D_{t}$ was found to have a value of $(1.6 \pm 0.4) \times 10^{-8} \mathrm{~cm}^{2} / \mathrm{s}$ at $30^{\circ} \mathrm{C}$. The exact dimensions of this protein are not known, but assumption of a membrane viscosity of $2 \mathrm{P}$ and the applicability of the Saffman-Delbrück model give the protein a radius of about $3.0 \mathrm{~nm}$, a value consistent with electron microscopic estimates of its size (Weinstein, 1974). From all of these results we conclude that the diffusion of large proteins at high dilution in liquid-crystalline phase lipid bilayers is in agreement with the model of Saffman and Delbrück (Saffman, 1976; Saffman $\&$ Delbrück, 1975). This conclusion will be more critically tested as proteins or protein aggregates with larger radii become accessible to experimental manipulation.

We now turn our attention to the diffusion of NBD-DMPE in the model membranes studied. We obtain values of $(8.8$ $\pm 1.3) \times 10^{-8},(1.4 \pm 0.1) \times 10^{-7}$, and $(1.1 \pm 0.1) \times 10^{-7} \mathrm{~cm}^{2} / \mathrm{s}$ for $D_{\mathrm{t}}$ of this probe in DMPC, total sarcoplasmic reticulum lipid, and soybean lipid multibilayers, respectively, all at about $37^{\circ} \mathrm{C}$. These values are in agreement with $D_{\mathrm{t}}$ for lipid diffusion reported by other workers with the FRAP technique (Wu et al., 1977; Smith et al., 1979b; Derzko \& Jacobson, 1980; Chang et al., 1981). For purposes of simplicity, we treat the lipid probe as a rigid cylindrical particle with a radius of $0.4 \mathrm{~nm}$ and a height of $2.5 \mathrm{~nm}$ (half the bilayer thickness). The model of Saffman \& Delbrück (1975; Saffman, 1976) with the "stick" assumption has been extended by Hughes et al. (1982) to include the case of diffusion, in viscous fluid sheets, of particles which experience nonequivalent and nonnegligible viscous drags at both ends of the particle. In this case $D_{\mathrm{t}}$ is given by

$$
D_{\mathrm{t}}=\frac{k T}{4 \pi \eta h}\left[\ln \left(\frac{2}{\epsilon}\right)-\gamma+\frac{4 \epsilon}{\pi}-\frac{\epsilon^{2}}{2} \ln \left(\frac{2}{\epsilon}\right)\right]
$$

where $\epsilon=\left(\eta_{1}{ }^{\prime}+\eta_{2}{ }^{\prime}\right) a /(\eta h)$. Here $\eta_{1}{ }^{\prime}$ and $\eta_{2}{ }^{\prime}$ are the viscosities of the bounding fluids on both sides of the viscous sheet (i.e., at both ends of the diffusing cylindrical particle embedded in the sheet) and are not necessarily equal to each other or negligible in comparison with $\eta$. The other symbols have the same meanings as before. A lipid molecule may be considered to be a cylinder diffusing in a lipid monolayer with $\eta$ (equal to the membrane viscosity) and the bounding fluid viscosities $\eta_{1}{ }^{\prime}$, equal to the viscosity of water, and $\eta_{2}{ }^{\prime}$, equal in the upper limit to $\eta$. In Figure 5 we plot calculated values of $D_{\mathrm{t}}$ as a function of $\eta_{2}^{\prime}$ for values of $h, a$, and $T$ of $2.5 \mathrm{~nm}, 0.4 \mathrm{~nm}$, and $310 \mathrm{~K}$, respectively. $\eta$ was taken at four values: $0.3,0.5,1.0$, and $2.0 \mathrm{P}$. The last two values were the extremes of membrane viscosities obtained from an analysis of the protein diffusion data (see above and Figure 4). It is seen from Figures 4 and 5 that the experimental values of lipid $D_{\mathrm{t}}$ are in agreement with the Saffman-Delbrück model as modified by Hughes et al. (1982) only when $\eta$ is less than $0.5 \mathrm{P}$ (with $\eta_{2}^{\prime} \approx \eta$ ). The results of time-resolved fluorescence anisotropy decay measurements using the hydrophobic probe 1,6-diphenyl-1,3,5hexatriene embedded in membranes are compatible with this value for $\eta$ (Kinoshita et al., 1981). Alternatively, if $\eta$ is in fact as high as it appears to be from the protein diffusion, the bilayer midplane viscosity, $\eta_{2}{ }^{\prime}$, must be less than $0.01 \mathrm{P}$. Fluorescence polarization studies of probes attached to the ends of fatty acid chains embedded in lipid bilayers have shown (Tilley et al., 1979; Vincent et al., 1982) that a nonzero "microviscosity" hinders their rotational motions. However, 


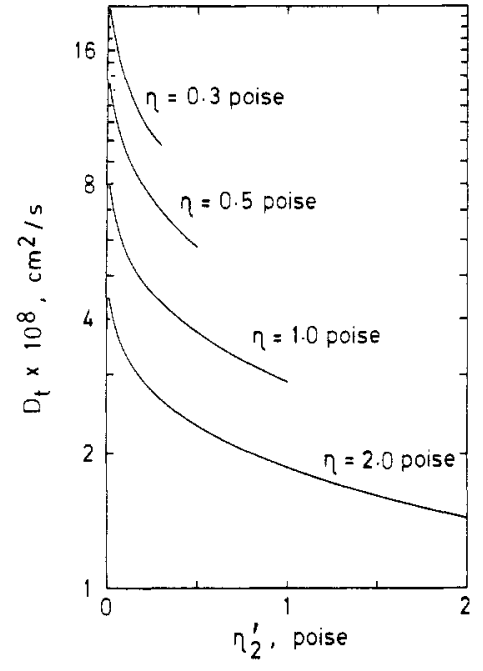

FIGURE 5: Predicted dependence of $D_{\mathrm{t}}$ for a lipid molecule upon the bilayer midplane viscosity, $\eta_{2}{ }^{\prime}$, at $310 \mathrm{~K}$ according to eq 2 . $h$ was assumed to be $2.5 \mathrm{~nm}$ (half the bilayer thickness), $a$ was assumed to be $0.4 \mathrm{~nm}$, and $\eta_{1}^{\prime}$ was assumed to be $0.01 \mathrm{P}$ (viscosity of water). The viscosity of the membrane was taken at values of $0.3,0.5,1.0$, and 2.0 P, and $\eta_{2}^{\prime}$ (the bilayer midplane viscosity) was varied from a minimum of $0.01 \mathrm{P}$ to a maximum of $\eta_{2}^{\prime}=\eta$ for each curve.

these probes are rather bulky and cannot be expected to give information about the degree of slip between the constituent monolayers of a lipid bilayer. In conclusion, at least three possible explanations of the lipid diffusion behavior may be presented: (1) The "stick" assumption may be valid for protein diffusion but not for lipid diffusion in bilayers. Depending on the degree of "slip" between the neighboring lipid molecules in a bilayer, the apparent viscosity felt by a lipid molecule as it translates in the plane of the membrane may be somewhat lower than that felt by large proteins. (2) The bilayer viscosity felt by proteins and lipids may be the same, but there may be a near-complete slip boundary condition at the bilayer midplane, i.e., the constituent monolayers slide over each other with no hindrance. (3) Different models may have to be invoked to explain lipid and protein diffusion in bilayers. We have recently shown (Vaz et al., 1982) that the experimentally obtained values of $D_{t}$ for small transbilayer peptides with radii about the same as that of a lipid molecule agree quite well with the predictions of the "free volume" diffusion model (Träuble \& Sackmann, 1972; Galla et al., 1979). If this is the case, it may be necessary to invoke a kinetic diffusion model for diffusants with diameters about equal to or less than that of the lipid and a continuum fluid hydrodynamic model for large diffusants. A similar proposal for diffusion in isotropic conditions has been made by Nir \& Stein (1971).

\section{Acknowledgments}

We gratefully acknowledge useful discussions with Drs. E. Matayoshi, R. Clegg, K. Jacobson, Z. Derzko, F. Jähnig, J. Stümpel, H. Füldner, and D. Marsh. We also thank Dr. W. Sawyer for having shown us a preprint of his paper (Hughes et al., 1982) and Dr. D. Hallmann for his gift of NBD-DMPE.

\section{References}

Albert, A. D., \& Litman, B. J. (1978) Biochemistry 17, 3893-3900.

Almeida, L. M., Vaz, W. L. C., Zachariasse, K. A., \& Madeira, V. M. C. (1982) Biochemistry (in press).
Axelrod, D., Koppel, D. E., Schlessinger, J., Elson, E., \& Webb, W. W. (1976) Biophys. J. 16, 1055-1069.

Brady, G. W., Fein, D. B., Harder, M. E., Spehr, R., \& Meissner, G. (1981) Biophys. J. 34, 13-34.

Chang, C. H., Takeuchi, H., Ito, T., Machida, K., \& Ohnishi, S. (1981) J. Biochem. (Tokyo) 90, 997-1004.

Cherry, R. J. (1979) Biochim. Biophys. Acta 559, 289-327.

Criado, M., Vaz, W. L. C., Barrantes, F. J., \& Jovin, T. M. (1982) Biochemistry (in press).

Derzko, Z., \& Jacobson, K. (1980) Biochemistry 19, 6050-6057.

Fleischer, S., Wang, C. T., Hymel, L., Seelig, J., Brown, M. F., Herbette, L., Scarpa, A., McLaughlin, A. C., \& Blasie, J. K. (1979) in Function and Molecular Aspects of Biomembrane Transport (Quagliariello, E., Ed.) pp 465-485, Elsevier/North-Holland Biomedical Press, Amsterdam.

Galla, H. J., Hartmann, W., Theilen, U., \& Sackmann, E. (1979) J. Membr. Biol. 48, 215-236.

Hughes, B. D., Pailthorpe, B. A., White, L. R., \& Sawyer, W. H. (1982) Biophys. J. 37, 673-676.

Jovin, T. M., Bartholdi, M., Vaz, W. L. C., \& Austin, R. H. (1981) Ann. N.Y. Acad. Sci. 366, 176-196.

Kinoshita, K., Kawato, S., Ikegami, A., Yoshida, S., \& Orii, Y. (1981) Biochim. Biophys. Acta 647, 7-17.

Madeira, V. M. C., \& Antunes-Madeira, M. C. (1976) Cienc. Biol. (Coimbra) 2, 265-291.

Nir, S., \& Stein, W. D. (1971) J. Chem. Phys. 55, 1598-1603.

Osborne, H. B., Sardet, C., Michel-Villaz, M., \& Chabre, M. (1978) J. Mol. Biol. 123, 177-206.

Peters, R., \& Cherry, R. J. (1982) Biophys. J. 37, $277 \mathrm{a}$.

Saffman, P. G. (1976) J. Fluid Mech. 73, 593-602.

Saffman, P. G., \& Delbrück, M. (1975) Proc. Natl. Acad. Sci. U.S.A. 72, 3111-3113.

Smith, L. M., Smith, B. A., \& McConnell, H. M. (1979a) Biochemistry 18, 2256-2259.

Smith, L. M., Parce, J. W., Smith, B. A., \& McConnell, H. M. (1979b) Proc. Natl. Acad. Sci. U.S.A. 76, 4177-4179.

Smith, L. M., Rubenstein, J. L. R., Parce, J. W., \& McConnell, H. M. (1980) Biochemistry 19, 5907-5911.

Tilley, L., Thulborn, K. R., \& Sawyer, W. H. (1979) J. Biol. Chem. 254, 2592-2594.

Träuble, H., \& Sackmann, E. (1972) J. Am. Chem. Soc. 94, 4499-4510.

Vaz, W. L. C., Jacobson, K., Wu, E. S., \& Derzko, Z. (1979) Proc. Natl. Acad. Sci. U.S.A. 76, 5645-5649.

Vaz, W. L. C., Kapitza, H. G., Stümpel, J., Sackmann, E., \& Jovin, T. M. (1981) Biochemistry 20, 1392-1396.

Vaz, W. L. C., Derzko, Z. I., \& Jacobson, K. A. (1982) Cell Surf. Rev. 8, 83-135.

Vincent, M., de Foresta, B., Gallay, J., \& Alfsen, A. (1982) Biochemistry 21, 708-716.

Weinstein, R. S. (1974) in The Red Blood Cell (Surgenor, D. M., Ed.) Vol. 1, pp 213-268, Academic Press, New York.

Wise, D. S., Karlin, A., \& Schoenborn, B. P. (1979) Biophys. J. 28, 473-496.

Wu, E. S., Jacobson, K., \& Papahadjopoulos, D. (1977) Biochemistry 16, 3936-3941.

Wu, E. S., Jacobson, K., Szoka, F., \& Portis, A. (1978) Biochemistry 17, 5543-5550.

Yeager, M. (1976) Brookhaven Symp. Biol. 27, 3-37.

Yeager, M., Schoenborn, B., Engelman, D., Moore, P., \& Stryer, L. (1980) J. Mol. Biol. 137, 315-348. 